\title{
Cytotoxic roles of apigenin and kaempferol on staurosporine-treated mesenchymal stem cells in an in vitro culture
}

\author{
MAGDALENA KOSIEDOWSKA ${ }^{\oplus}$, ARKADIUSZ BURCZAK ${ }^{\circledR}$, JULIA MORYS*๑, \\ PAULINA BORKOWSKA॰ ${ }^{\circledR}$ JAN KOWALSKI॰
}

Department of Medical Genetics

Medical University of Silesia

Jedności 8

41-200 Sosnowiec, Poland

*corresponding author: phone: +48 32364 1216; e-mail: julia.morys@sum.edu.pl

\section{Summary}

Introduction: Flavonoids are widely distributed in the wild. They constitute a large group of compounds that have a beneficial effect on the human body. Apigenin and kaempferol, which belong to the flavone subgroup, have, inter alia, an antitumor effect. The influence of these compounds on the survival of stem cells in a toxic environment has not yet been studied.

Objective: The aim of the study was to evaluate the effect of selected concentrations of apigenin and kaempferol on the survival of mesenchymal stem cells (MSC) in the presence of a cell-death inducer - staurosporine.

Methods: Mesenchymal stem cells that were obtained from the Wharton's jelly of umbilical cords were used for the research. In the first stage, the MSC were treated with apigenin at concentrations of 1.2, 12.5, 25, 50 and $100 \mu \mathrm{M} / \mathrm{ml}$ and kaempferol at concentrations of $1.2,12.5,25,50$ and $100 \mu \mathrm{M} / \mathrm{ml}$. In the next stage, the effect of increased concentrations of $0.1,0.5$ and $1 \mu \mathrm{M} / \mathrm{ml}$ of staurosporine on MSC was examined. The key stage of the experiment was investigating the interaction between the selected concentrations of apigenin $(12.5$ and $50 \mu \mathrm{M} / \mathrm{ml})$ and kaempferol $(12.5$ and $\mu \mathrm{M} / \mathrm{ml})$ on MSC in the presence of staurosporine at a concentration of $1 \mu \mathrm{M} / \mathrm{ml}$, which had the highest toxicity.

Results: Both apigenin and kaempferol significantly increased the cytotoxic features of staurosporine on the MSC culture.

Key words: apigenin, kaempferol, staurosporine, cytoprotection, cytotoxicity, mesenchymal stem cells

Słowa kluczowe: apigenina, kemferol, staurosporyna, cytoprotekcja, cytotoksyczność, mezenchymalne komórki macierzyste 


\section{INTRODUCTION}

Flavonoids are a group of chemical compounds whose common denominator is their presence and chemical structure. They are found in numerous plants, including algae and bryophytes. They are present in greatest amounts in flowers and leaves and give them a yellowish colour. All flavonoids have the basic 15-carbon structure of 2-phenylchroman that forms two phenyl rings and a heterocyclic ring that contains oxygen - so-called C6-C3-C6 structure. Several thousand flavonoid compounds are known. Because of the differences in the number and location of the hydroxyl groups, the presence of a ketone group in the heterocyclic ring, the type of glycosidic bonds as well as the number and characteristics of the attached sugar particles, there are subgroups of flavonoids, which include, among others, flavones, flavonols, flavanones, isoflavones, catechins and anthocyanidins [1].

An example of a substance that belongs to the subgroup of flavones is apigenin (4, 5,7-trihydroxy flavone with a molecular weight of $270.24 \mathrm{~g} / \mathrm{mol}$ ). It is a relatively small chemical compound that is practically insoluble in water $(0.00135 \mathrm{mg} / \mathrm{ml})$, which is found mainly in parsley leaves (approx. 13,500 mg/100 g, i.e., $500 \mu \mathrm{M} / \mathrm{g})$, chamomile $(40-740 \mathrm{mg} / 100$ g, i.e., $1.5-27.4 \mu \mathrm{M} / \mathrm{g}$ ) as well as in propolis (approx. $500 \mathrm{mg} / 100$ g, i.e., $18.5 \mu \mathrm{M} / \mathrm{g}$ and in a smaller amount in celery (approx. $19 \mathrm{mg} / 100$ g, i.e., $70 \mu \mathrm{M} / 100 \mathrm{~g}$ ), St. John's wort and other plants [2].

The activity of apigenin includes inducing cell cycle arrest, inhibiting cell migration and stimulating the immune response [3]. In addition, apigenin also has an anti-inflammatory effect $[4,5]$, removes free radicals and activates the enzymes of the phase II metabolism. In studies conducted in mice, it was proven that it has an anti-atherosclerotic activity by regulating the metabolism of the low-density lipoproteins (LDL) [6]. Insulin secretion studies in mice also showed that apigenin improves the regenerative capabilities in diabetic cardiomyopathy and kidney dysfunction. Clinical trials and studies on animal models have also been conducted on the therapeutic potential of apigenin as a neuroprotective factor for treating diseases such as depression, Alzheimer's disease or Parkinson's disease [7].

The representative of the flavonol subgroup is kaempferol (3,4', 5,7-tetrahydroxy flavone). Kaempferol is a yellow-coloured organic chemical of a low molecular weight $(286.2 \mathrm{~g} / \mathrm{mol})$. It belongs to the group of flavonoids and has hydroxyl groups in its structure. It is a compound that is widespread in the plant kingdom. It is most abundant in leek leaves (approx. $50 \mathrm{mg} / 100 \mathrm{~g}$, i.e., $175 \mu \mathrm{M} / 100 \mathrm{~g}$ ) and in chicory (approx. $24 \mathrm{~g} / 100 \mathrm{~g}$, i.e., $84 \mu \mathrm{M} / 100 \mathrm{~g}$ ) [8, 9]. It is also present in smaller amounts in tea leaves, cabbage, kale, broccoli and beans as well as in tomatoes, grapes and strawberries. Kaempferol represents antioxidant properties performed by reduction of reactive oxygen species. This effect is exhibited by enhancing the activity of catalase and superoxide dismutase. Kaempferol also has antibacterial and anti-atherosclerotic features. In the studies of many scientists, the neuroprotective and antitumor effects of kaempferol have been also proven by its inhibiting angiogenesis and inducing apoptosis $[10,11]$.

Staurosporine is a natural antibiotic originally isolated from Streptomyces staurosporeus [12], wellknown for its features such as nonselective inhibition of a variety of protein kinases [12-14]. Some of its derivatives are often used in cancer therapy [12]. Recent studies have confirmed that its death inducing mechanism includes apoptosis as well as necrosis. However, the signalling pathway remains questionable [15].

The aim of this study was to investigate the influence of apigenin and kaempferol on the survival of mesenchymal stem cells (MSC) in the toxic conditions that are induced by the action of staurosporine.

\section{MATERIALS AND METHODS}

\section{Isolation, culture and characterisation of WJ-MSC}

Mesenchymal stem cells were isolated from the Wharton's jelly of the umbilical cords of women after their delivery, according to a previously published protocol [16]. The study was approved by the Bioethics Committee of the Medical University of Silesia (approval no. KNW/0022/KB/195/14). The umbilical cords were collected from the Department of Obstetrics and Gynaecology with the Subdivision of Oncological Gynaecology, Brothers Hospitallers of Saint John of God Hospital in Katowice, Poland. After having read the test procedure, the participants gave their written consent to use the umbilical cords. The homogeneity of the cell culture was quantified using flow cytometry. The presence of any antigens on the cell surface was also determined: CD73, CD90, CD34, CD11b, CD19, CD45 and HLA-DR. The MSC were characterized by their ability to differentiate into adipocytes and osteocytes. All the MSC characterization procedures have been published previously [16]. 


\section{Cell culture}

The cultured cells were plated in 96-well plates at 5,000 cells per well. The cultivation was conducted in the following medium: DMEM/F12 (PAN Biotech; P04-41250), 15\% FBS (PAN Biotech; P30$8500)$ and a $1 \%$ antibiotic antimycotic solution (100X) (PAN Biotech; P06-07300). The prepared plates were incubated overnight at $37^{\circ} \mathrm{C}$. Then, the culture medium was removed and the medium with an amount of FBS reduced to $2 \%$ was used. The next stage of the research was selecting the optimal concentrations of the tested substances. Apigenin was used successively at concentrations of $[\mu \mathrm{M} / \mathrm{ml}] 1.2$; $12.5 ; 25 ; 50$ and 100 and kaempferol at concentrations of $[\mu \mathrm{M} / \mathrm{ml}] 1.2 ; 12.5 ; 25,50$ and 100 . Staurosporine (Sigma-Aldrich; S4400) was added to the third 96-well MSC plate in order to determine the concentration of staurosporine that induced apoptosis most strongly at concentrations of $[\mu \mathrm{M} / \mathrm{ml}]$ $0.1 ; 0.5$ and 1 . A key step in the experiment was the addition of the selected concentrations of apigenin $(12.5 \mu \mathrm{M} / \mathrm{ml}$ and $50 \mu \mathrm{M} / \mathrm{ml})$ or kaempferol $(12.5 \mu \mathrm{M} / \mathrm{ml}$ and $50 \mu \mathrm{M} / \mathrm{ml})$ to the MSC and then (after 2 hours) $1 \mu \mathrm{M}$ of staurosporine. The experiment was designed in this way in order to determine whether apigenin and kaempferol have cytoprotective effects that can protect cells against death in the stress and toxic conditions that are induced by staurosporine.

\section{Cell viability in WST-1 colorimetric assay}

After incubation with apigenin or kaempferol in a staurosporine medium for 24 hours, the culture medium was removed and $100 \mu \mathrm{l}$ of a 10\% WST-1 reagent solution (Roche; 11644807001) in PBS was added to each well of the plates. The plates were incubated for 45 minutes at $37^{\circ} \mathrm{C}$ after which the extinction was read at $450 \mathrm{~nm}$ using an ELX $800 \mathrm{IU}$ automatic microplate reader (Bio-Tek Instruments, Inc., Winooski, VT, USA) and then Bio-Tek Gene 5 Software V 3.02 was used to analyze the results. The experiment was performed in four wells for each substance and repeated three times in independent experiments. The sample absorbance values after subtracting the background absorbance values were used to calculate cell survival. The surviving cell fraction was determined by comparing it with the negative control cells (MSC that had been grown under the same conditions but that had not been treated with any of the substances).

\section{Statistical analysis}

The points represent the mean value $\pm S D(n=12$; three independent experiments). Statistically significant ${ }^{\star} p<0.05$. GraphPad Prism software version 8.0 was used to analyse the data. The Student's t-test was also performed.

\section{RESULTS}

At first, the toxic staurosporine conditions to obtain the required environment had to be established. To optimize the dosage of staurosporine that generates the toxic conditions in an MSC culture, the effect of staurosporine at concentrations of $0.1,0.5$ and $1 \mu \mathrm{M} / \mathrm{ml}$ for MSC survival was studied. When used at a concentration of $0.5 \mu \mathrm{M} / \mathrm{ml}$, staurosporine reduced the survival by $20 \%$ and at a dose of $1 \mu \mathrm{M} / \mathrm{ml}$ by $50 \%$ compared to the control group (fig. 1). Staurosporine at a dose of $1 \mu \mathrm{M} / \mathrm{ml}$ was selected for further research because it was effective in generating a toxic environment which, to some extent, reflected the toxic conditions in the brain in the course of neurodegenerative diseases.



Figure 1.

Survival rate of the MSC in the presence of the selected concentrations of staurosporine. The points represent the mean value $\pm S D$ ( $n=12$; three independent experiments). Statistically significant ${ }^{\star} p<0.05$; Student's t-test.

The low dose of apigenin, $1.2 \mu \mathrm{M} / \mathrm{ml}$, did not affect the survival of the MSC. When apigenin was added to the MSC cultures at doses of $12.5 \mu \mathrm{M} / \mathrm{ml}$ and higher, it had a statistically significant dose-dependent cytotoxic effect. At a dose of $12.5 \mu \mathrm{M} / \mathrm{ml}$ apigenin, the survival of the MSC was $20 \%$ lower than in the control group, while at 
doses in the range of $25-100 \mu \mathrm{M} / \mathrm{ml}$, the survival of the MSC was $50 \%$ lower than in the control group. Adding kaempferol to the MSC cultures at doses ranging from $1.2-25 \mu \mathrm{M} / \mathrm{ml}$ did not affect the survival of the MSC. In turn, the doses of 50 and $100 \mu \mathrm{M} / \mathrm{ml}$ resulted in a statistically significant reduction in their survival by $20 \%$ compared to the control group (fig. 2).

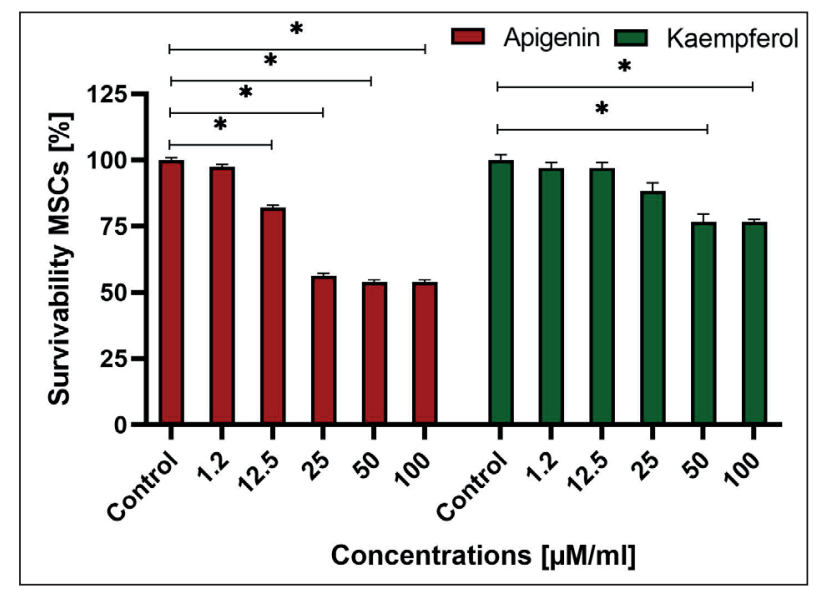

Figure 2.

Survival rate of the MSC at increasing concentrations of apigenin and kaempferol. The points represent the mean value $\pm S D(n=12$; three independent experiments). Statistically significant ${ }^{*} p<0.05$; Student's t-test.

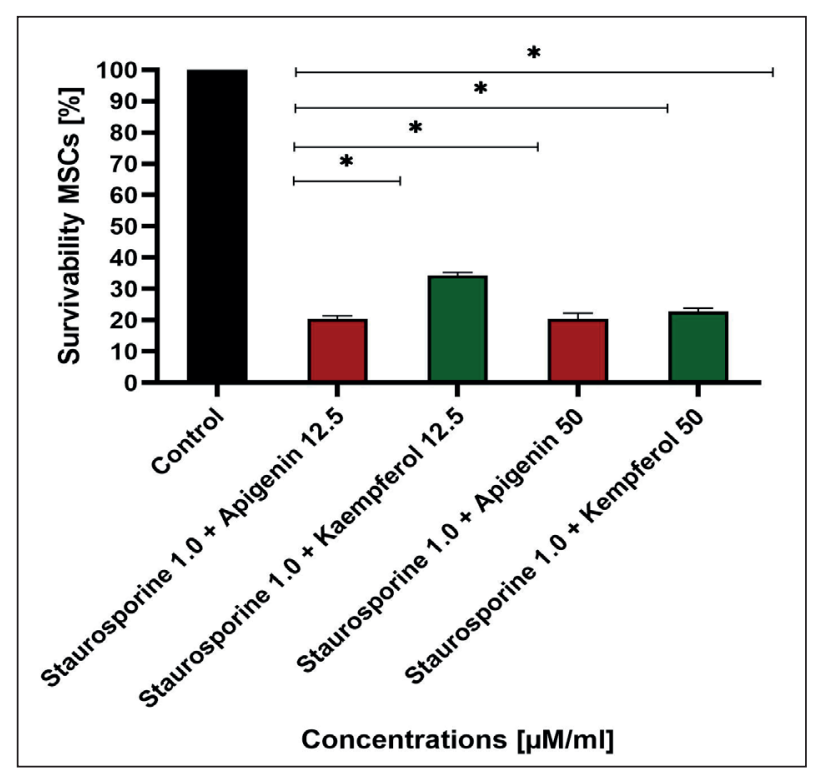

Figure 3.

Survival rate of the MSC in the presence of staurosporine after the addition of apigenin at concentrations of 12.5 $\mu \mathrm{M} / \mathrm{ml}$ and $50 \mu \mathrm{M} / \mathrm{ml}$ or kaempferol at concentrations of $12.5 \mu \mathrm{M} / \mathrm{ml}$ and $50 \mu \mathrm{M} / \mathrm{ml}$. The points represent the mean value $\pm S D(n=12$; three independent experiments). Statistically significant ${ }^{*} p<0.05$; Student's t-test.
Apigenin and kaempferol at doses of 12.5 and $50 \mu \mathrm{M} / \mathrm{ml}$ were selected for the study of the effect of the substances on the survival of MSC under toxic conditions. After the apigenin or kaempferol was used, the cytotoxic effect of the substance increased for each dose and the relationship was statistically significant. Additionally, we observed an additive effect of the cytotoxic effect of the tested substances and the toxic effect of staurosporine (fig. 3).

\section{DISCUSSION}

Both apigenin and kaempferol have been extensively studied for their anti-cancer $[3,10,11]$, anti-inflammatory $[4,5]$ and anti-atherosclerotic effects $[6,17$, 18]. At the same time, the study of the influence of the analysed substances on MSC breeding is a pioneering study, considering the fact that based on the results that have been obtained by other authors [19-22], flavonoids are considered to be bioactive compounds that have neuroprotective properties. The conducted study proved the potential cytotoxicity of apigenin and kaempferol, which is in contrast to the results of the research that has been published to date. This may be caused by the higher sensitivity of the MSC that were used in this study compared to the differentiated neuronal cells that have been tested by other research units as well as by the use of staurosporine in the experiment.

To some extent, staurosporine mimics the conditions in the brain in the course of neurodegenerative diseases. The additive cytotoxic effect of staurosporine and apigenin or kaempferol calls into question the usefulness of these substances in prevention or treatment of neurodegenerative diseases, which has been suggested by other authors $[7,23]$.

Research on the synergistic effects of various flavonoids is still ongoing. There are reports that have confirmed the mutual potentiation of the activity of the compounds from this group [24]. Therefore, it is necessary to take into account the elimination of harmful bioactive substances in certain diseases. Based on the results of this experiment, the consumption of apigenin and kaempferol should be limited in individuals with an increased likelihood of developing neurodegenerative diseases. The deficit that results from their removal should be supplemented with neuroprotective flavones, which would increase the demand for these compounds, and thereby reduce the risk of the increased incidence of neurodegenerative diseases. 
The created test model using staurosporine, to some extent reflects the toxic environment in the brains of patients suffering from neurodegenerative diseases such as Alzheimer's, Parkinson's, Huntington's diseases or amyotrophic lateral sclerosis (ALS). Therefore, we recommend that the consumption of products that are sources of apigenin and kaempferol should be limited by people who are at risk or in early stages of such diseases progression in order to prevent its progress in the future.

\section{CONCLUSIONS}

a) In vitro, apigenin and kaempferol are cytotoxic to MSC and MSC that have been exposed to staurosporine

and

b) a synergistic cytotoxic effect of staurosporine and apigenin or staurosporine and kaempferol on the culture of MSC was observed.

\section{ACKNOWLEDGEMENTS}

This work was supported by a grant from the Medical University of Silesia, Poland (KNW-1-064/N/9/B). The authors would like to thank prof. Rafał Stojko $\mathrm{MD}, \mathrm{PhD}$ for collecting the umbilical cords.

Conflict of interest: Authors declare no conflict of interest.

\section{REFERENCES}

1. Shashank K, Abhay P. Chemistry and biological activities of flavonoids: an overview. Sci World J 2013; 162750. doi: https://dx.doi. org/110.1155/2013/162750

2. Haghi G, Hatami A, Safaei A, Mehran M. Analysis of phenolic compounds in Matricaria chamomilla and its extracts by UPLC-UV. Res Pharm Sci 2014; 9(1):31-37.

3. Yan X, Qi M, Li P, Zhan Y, Shao H. Apigenin in cancer therapy: anti-cancer effects and mechanisms of action. Cell Biosci 2017; 7(1). doi: https://dx.doi.org/110.1186/s13578-017-0179-x

4. Lee J-H, Zhou H J, Cho S Y, Kim Y S, Lee Y S, Jeong Ch S. Anti-inflammatory mechanisms of apigen- in: inhibition of cyclooxygenase-2 expression, adhesion of monocytes to human umbilical vein endothelial cells and expression of cellular adhesion molecules. Arch Pharm Res 2007; 30(10): 13181327. doi: http://dx.doi.org/10.1007/BF02980273

5. Kadioglu O, Nass J, Saeed M E M, Schuler B, Efferth T. Kaempferol is an anti-inflammatory compound with activity towards NF- $\kappa$ B pathway proteins. Anticancer Res 2015; 35(5):2645-50.

6. Fahad AR, Falaq N, Smita J, Yasir HS. Health functionality of apigenin: a review. Int J Food Prop 2017; 20:1197-1238. doi: https://dx.doi.org /110.1080/10942912.2016.1207188

7. Salehi B, Venditti A, Sharifi-Rad M, Kręgiel D, Sharifi-Rad J, Durazzo A et al. The therapeutic potential of apigenin. Int J Mol Sci 2019; 20(6):1305. doi: http://dx.doi.org/10.3390/ijms20061305

8. DuPont MS, Day AJ, Bennett RN, Mellon FA, Kroon PA. Absorption of kaempferol from endive, a source of kaempferol-3-glucuronide, in humans. Eur J Clin Nutr 2004; 58(6):947-954. doi: http://dx.doi.org/10.1038/sj.ejcn.1601916

9. Dabeek WM, Marra MV. Dietary quercetin and kaempferol: bioavailability and potential cardiovascular-related bioactivity in humans. Nutrients 2019; 11(10):2288. doi: http://dx.doi.org/10.3390/ nu11102288

10. Imran M, Rauf A, Shah ZA, Saeed F, Imran A, Arshad MU. Chemo-preventive and therapeutic effect of the dietary flavonoid kaempferol: a comprehensive review. Phytother Res 2018; 1(13):113. doi: http://dx.doi.org/10.1002/ptr.6227

11. Ulanowska M, Olas B. Fitozwiązki - ważne składniki suplementów diety oraz ich wpływ na zdrowie człowieka. Kosmos. 2021; 70(1):103-114. doi: https://dx.doi.org/110.36921/kos.2021_2721

12. Malsy M, Bitzinger D, Graf B, Bundscherer A. Staurosporine induces apoptosis in pancreatic carcinoma cells PaTu 8988t and Panc-1 via the intrinsic signaling pathway. Eur J Med Res 2019; 24: 5. doi: http://dx.doi.org/10.1186/s40001-0190365-x

13. McCarthy MJ, Rubin LL, Philpott KJ. Involvement of caspases in sympathetic neuron apoptosis. J Cell Sci 1997; 110(Pt 18):2165-73. 
14. Koh JY, Wie MB, Gwag BJ, Sensi SL, Canzoniero LM, Demaro J et al. Staurosporine-induced neuronal apoptosis. Exp Neurol 1995; 135(2):153-9. doi: http://dx.doi.org/10.1006/exnr.1995.1074

15. Linares GR, Leng Y, Maric D, Chuang D-M. Overexpression of fibroblast growth factor-21 (FGF21) protects mesenchymal stem cells against caspase-dependent apoptosis induced by oxidative stress and inflammation. Cell Biol Int 2020; 44:2163-2169. doi: http://dx.doi.org/10.1002/ cbin.11409

16. Borkowska P, Zielinska A, Paul-Samojedny M, Stojko R, Kowalski J. Evaluation of reference genes for quantitative real-time PCR in Wharton's Jelly-derived mesenchymal stem cells after lentiviral transduction and differentiation. Mol Biol Rep 2020; 47(2):1107-1115. doi: http:// dx.doi.org/10.1007/s11033-019-05207-6

17. Gao H-L, Yu X-J, Hu H-B, Yang Q-W, Liu K-L, $\mathrm{Chen} \mathrm{Y}-\mathrm{M}$ et al. Apigenin improves hypertension and cardiac hypertrophy through modulating NADPH oxidase-dependent ROS generation and cytokines in the hypothalamic paraventricular nucleus. Cardiovascular Toxicology 2021; 21:721736. doi: http://dx.doi.org/10.1007/s12012-02109662-1

18. Ciumărnean L, Milaciu M V, Runcan $\mathrm{O}$, Vesa S C, Răchis A L, Negrean V et al. The effects of flavonoids in cardiovascular diseases. Molecules 2020; 25, 4320. doi: http://dx.doi.org/10.3390/ molecules 25184320

19. Dourado N S, Souza C S, Almeida M M A, Silva A B, Santos B L, Silva V D A et al. Neuroimmunomodulatory and neuroprotective effects of the flavonoid apigenin in in vitro models of neuroinflammation associated with Alzheimer's disease. Front Aging Neurosci 2020; 12:119. doi: https:// dx.doi.org/110.3389/fnagi.2020.00119
20. Zhao L, Wang J-L, Liu R, Li X-X, Li J-F, Zhang L. Neuroprotective, anti-amyloidogenic and neurotrophic effects of apigenin in an Alzheimer's disease mouse model. Molecules 2013; 18 : 9949-9965. doi: https://dx.doi.org/110.3390/molecules 18089949

21. Paria H, Javad F B, Somaye V, Farnaz N Evaluation of the neuroprotective, anticonvulsant and cognition improvement effects of apigenin in temporal lobe epilepsy: Involvement of the mitochondrial apoptotic pathway. Iranian Journal of Basic Medical Sciences 2019; 22(7): 752-758. doi: http:// dx.doi.org/10.22038/ijbms.2019.33892.8064

22. Vauzour D, Vafeiadou K, Rodriguez-Mateos A, Rendeiro C, Spencer J P E. The neuroprotective potential of flavonoids: a multiplicity of effects. 2008; 3(3-4): 115-126. doi: http://dx.doi. org/10.1007/s12263-008-0091-4

23. de Araújo FF, de Paulo Farias D, Neri-Numa IA, Pastore GM. Polyphenols and their applications: An approach in food chemistry and innovation potential. Food Chem 2021; 15;338:127535. doi: http://dx.doi.org/10.1016/j. foodchem.2020.127535

24. Ahn-Jarvis J H, Parihar A, Doseff A I. Dietary flavonoids for immunoregulation and cancer: food design for targeting disease. Antioxidants 2019; 8(7): 202. doi: http://dx.doi.org/10.3390/ antiox 8070202 